\title{
Pancreatic neuroendocrine tumors: correlation between histogram analysis of apparent diffusion coefficient maps and tumor grade
}

\author{
Jose Antonio Sousa Pereira, ${ }^{1}$ Elsa Rosado, ${ }^{2}$ Maria Bali, ${ }^{3}$ Thierry Metens, ${ }^{3}$ Shih-Li Chao ${ }^{4}$ \\ ${ }^{1}$ Department of Radiology, Centro Hospitalar de Lisboa Central, Lisbon, Portugal \\ ${ }^{2}$ Department of Radiology, Hospital Fernando Fonseca, Amadora, Portugal \\ ${ }^{3}$ Department of Radiology, Hôpital Erasme, Université Libre de Bruxelles, Brussels, Belgium \\ ${ }^{4}$ Department of Radiology, Institut Jules Bordet, Université Libre de Bruxelles, Brussels, Belgium
}

\begin{abstract}
Purpose: To explore the role of histogram analysis of apparent diffusion coefficient (ADC) MRI maps based on entire tumor volume data in determining pancreatic neuroendocrine tumor (PNT) grade.

Methods and Materials: Retrospective evaluation of 22 patients with PNTs included low-grade (G1;n=15), intermediate-grade $(\mathrm{G} 2 ; n=4)$, and high-grade $(\mathrm{G} 3$; $n=3$ ) tumors. Regions of interest containing the lesion were drawn on every section of the ADC map containing the tumor and summated to obtain histograms for entire tumor volume. Calculated histographic parameters included mean ADC (mADC), 5th percentile ADC, 10th percentile ADC, 25th percentile ADC, 50th percentile ADC, 75th percentile ADC (ADC75), 90th percentile ADC (ADC90) and 95th percentile ADC (ADC95), skewness and kurtosis. Histogram parameters were correlated with tumor grade by repeated measures analysis of variance with Tukey-Kramer post hoc comparisons.

Results: The mADC, ADC75, ADC90, and ADC95 were significantly higher in G1 tumors $(1283 \pm 267$; $\left.1404 \pm 300 ; \quad 1495 \pm 318 ; \quad 1562 \pm 347 \times 10^{-6} \mathrm{~mm}^{2} / \mathrm{s}\right)$ compared to $\mathrm{G} 2(892 \pm 390 ; 952 \pm 381 ; 1036 \pm 384$; $\left.1072 \pm 374 \times 10^{-6} \mathrm{~mm}^{2} / \mathrm{s}\right)$ and to G3 tumors $\left(733 \pm 225 ; 864 \pm 284 ; 1008 \pm 288 ; 1152 \pm 192 \times 10^{-}\right.$ $\left.{ }^{6} \mathrm{~mm}^{2} / \mathrm{s}\right)(p$ value $<0.05)$. Skewness and kurtosis were significantly different between G1 $(0.041 \pm 0.466$; $2.802 \pm 0.679)$ and $\mathrm{G} 3(1.01 \pm 1.140 ; 5.963 \pm 4.008)$ tumors ( $p$ value $<0.05)$. Tumor volume $(\mathrm{mL})$ was significantly higher on G3 (55 \pm 15.7$)$ compared to G1
\end{abstract}

Correspondence to: Jose Antonio Sousa Pereira; email: pereirajoseantonio@outlook.com
$(1.9 \pm 2.7)$ and $\mathrm{G} 2(4.5 \pm 3.6)$ tumors $(p$ value $<0.05)$. In this small sample size, we did not detect statistically significant parameters between $\mathrm{G} 2(n=4)$ and G3 $(n=3)$ tumors.

Conclusions: Histographic analysis of ADC maps on the basis of the entire tumor volume can be useful in differentiating histologic grades of PNTs.

Key words: Pancreatic - Neuroendocrine-

Histogram-ADC

Pancreatic neuroendocrine tumors (PanNET) are a rare group of heterogeneous tumors which represent only 1$2 \%$ of all pancreatic neoplasms [1] and have an estimated incidence rate in several population studies of $<1 \%$ [2]. However, in the last 20 years, its incidence has increased and this is thought to be due to increased awareness, improved diagnostic imaging modalities, and a revision of the definition $[1,3,4]$.

In 2010, the World Health Organization (WHO) adopted a grading system (G1, G2, and G3) based on the mitotic count per square millimeter of tumor and the Ki67 index, that was created by the European Neuroendocrine Tumor Society (ENETS) [5, 6] (Table 1). In this system, well-differentiated PanNET (WDNET) are divided into low grade (G1) and intermediate grade $(\mathrm{G} 2)$, while the high grade (G3) refers to poorly differentiated neuroendocrine carcinoma (PDNEC). Studies have shown the usefulness of this grading system, as some of the well-differentiated tumors with higher proliferative index (G2) seem to have worse prognosis than PanNET with lower proliferative index (G1) [6, 7]. It has been shown that this grading system is a valid tool for prog- 
nostic stratification in clinical practice and research $[8,9]$. The choice of treatment depends not only on the symptoms, stage of disease, degree of uptake of radionuclide but also on tumor grade [10]. For example, treatment with somatostatin analogs are indicated only in $\mathrm{G} 1 / \mathrm{G} 2$ tumors, therefore, pre-treatment grading of the tumor is considered essential [10].

Magnetic resonance (MR) imaging is one of the recommended imaging modalities for the detection of suspected PanNET [10]. Diffusion-weighted MR imaging (DW-MRI) allows non-invasive assessment of biological tissues based on the molecular motion of water molecules in the extracellular, intracellular, and the intravascular spaces. In tissues, such as tumors characterized by high cellular density, increased tortuosity of the extracellular space and high density of hydrophobic cellular membranes restricted diffusion is seen [11-13].

Apparent diffusion coefficient (ADC) maps have been used to characterize tumoral tissues. These maps provide a quantitative index of water diffusivity for each voxel, allowing visualization of molecular diffusion in the different tissue compartments and providing indirect information on its microstructure [11]. Recently, there has been growing interest in the histogram analysis of ADC of the entire tumor volume, as this approach provides quantitative information on the distribution and frequency of ADCs and consequently on the heterogeneity of water molecules diffusivity within the whole tumor [14-17]. The histogram-based ADC analysis has shown capabilities in differentiating and grading brain tumors [14-16], and in the prediction of uterine cervical cancer recurrence [18, 19].

Previous studies have evaluated the correlation of ADC with histopathologic characteristics of PanNET [12, 13, 20]. However, these studies were based on regions of interest (ROI) placed on a representative section of the tumor, which does not reflect in detail the histological features of the tumor, something that might be paramount for PanNET, as treatment choice depends on these features [10].

To our knowledge, there are no previous reports of volume-based histogram analysis of ADC to differentiate PanNET grades. Therefore, the purpose of this study was to assess if histogram analysis of ADC from the entire tumor volume allows differentiation of the histological grades of PanNETs.

\section{Materials and methods}

This retrospective study was approved by the institutional review board and a waiver of informed consent was obtained.

\section{Study population}

From the clinical records of our institution, we identified 33 patients with histological proven PanNET who underwent abdominal MR imaging between 2007 and 2012.
The inclusion criteria consisted of MR examinations including DW-MR performed within a delay of 30 days before surgery.

Nine patients were excluded because MR was not performed before surgery. Two patients were excluded because the PanNET was intra-ductal. Finally, 22 patients were included: 19/22 (86\%) had PanNET histological confirmation after surgical excision and $3 / 22$ (14\%) had histological confirmation based on endoscopic biopsy samples ( 2 patients did not undergo surgery due to the presence of metastatic disease at the diagnosis and 1 patient refused surgery).

\section{$M R$ imaging technique}

Among the 22 patients, 18/22 (82\%) were investigated on a 1.5T magnet (Achieva, Philips Health System, Best, The Netherlands) and 4/22(16\%) patients were scanned on a 3T magnet (Achieva Multitransmit, Philips Health System, Best, The Netherlands). In both systems, a 16 channel received coil was used. All patients were imaged after $4 \mathrm{~h}$ fasting in the supine position. The MR imaging protocol included the following pulse sequences: (a) axial and coronal single-shot turbo spin-echo (TSE) T2-weighted images covering the upper abdomen were acquired with respiratory triggering $(\mathrm{TE}=80 \mathrm{~ms}$, Echo train length: 72, 40 slices of $5 \mathrm{~mm}$ thickness and no interslice gap and a field of view of $350 \times 400 \mathrm{~mm}$ ), (b) axial spin-echo echo planar DW-MRI sequence acquired with respiratory triggering, (TE $70 \mathrm{~ms}$, Echo train length: 61, 40 slices of $5 \mathrm{~mm}$ section thickness, no interslice gap, field of view of $350 \times 400 \mathrm{~mm}$ ). Diffusion-probing gradients were applied in three orthogonal directions and the isotropic mean image was obtained. The DW-MRI sequence was acquired with $\mathrm{b}$ factors 0,150 , and $1000 \mathrm{~s} / \mathrm{mm}^{2}$. All sequences were acquired with a sense acceleration factor of 2 .

\section{Histological analysis}

Histological evaluation was made on surgically resected specimens in 19/22 patients and on endoscopic biopsy samples in $3 / 22$ patients. Grading classification was based on mitotic count per square millimeter of tumor and $\mathrm{Ki}-67$ index, according to the ENETS $[5,6]$ adopted by the WHO in 2010 (Table 1).

\section{Image Analysis}

Two radiologists (one third year resident in radiology and one radiologist with 16 years of experience in abdominal MR) analyzed all images, working in consensus and blinded to histological grading of the PanNET. Data from all bvalues were transferred from a picture archiving and communication system (PACS) to a personal computer. Using an in-house developed software, an ADC map was calculated for each slice from images with $b$ values $\geq 150 \mathrm{~s} / \mathrm{mm}^{2}$, 
Table 1. Classification system for pancreatic neuroendocrine tumors

\begin{tabular}{llll}
\hline Differentiation & Grade & Mitoses/10|HPF & Ki-67 proliferation|index (\%) \\
\hline Well-differentiated PanNET & Grade 1 & $<2$ & $<3$ \\
Well-differentiated PanNET & Grade 2 & $2-20$ & $3-20$ \\
Poorly differentiated PanNEC & Grade 3 & $>20$ & $>20$ \\
\hline
\end{tabular}

$H P F$ high power field, PanNET pancreatic neuroendocrine tumor, PanNEC pancreatic neuroendocrine carcinoma

Table 2. Histogram-derived parameters (mean \pm SE)

\begin{tabular}{|c|c|c|c|c|c|c|c|c|c|c|}
\hline & $\mathrm{mADC}$ & ADC5 & ADC10 & ADC25 & ADC50 & ADC75 & ADC90 & ADC95 & Skew & Kurtosis \\
\hline G1 & $1283 \pm 267$ & & & & & & & & $0.041 \pm 0.466$ & $2.802 \pm 0.679$ \\
\hline G2 & $892 \pm 390$ & $664 \pm 420$ & $716 \pm 409$ & $800 \pm 390$ & $880 \pm 385$ & $952 \pm 381$ & $1036 \pm 384$ & $1072 \pm 374$ & $0.065 \pm-0.387$ & $3.788 \pm 1.220$ \\
\hline G3 & $733 \pm 225$ & $448 \pm 254$ & $512 \pm 284$ & $608 \pm 284$ & $725 \pm 272$ & $864 \pm 284$ & $1008 \pm 288$ & $1152 \pm 192$ & $1.01 \pm 1.140$ & $5.963 \pm 4.008$ \\
\hline
\end{tabular}

Table 3. Significant differences in ANOVA and post hoc comparisons ( $p$ values)

\begin{tabular}{lcccccccccc}
\hline & mADC & ADC5 & ADC10 & ADC25 & ADC50 & ADC75 & ADC90 & ADC95 & Skew & Kurtosis \\
\hline G1vsG2 & $\mathbf{0 . 0 4 9}$ & 0.208 & 0.185 & 0.104 & 0.069 & $\mathbf{0 . 0 3 4}$ & $\mathbf{0 . 0 3 8}$ & $\mathbf{0 . 0 2 9}$ & 0.941 \\
G1vsG3 & $\mathbf{0 . 0 0 4}$ & $\mathbf{0 . 0 1 9}$ & $\mathbf{0 . 0 1 7}$ & $\mathbf{0 . 0 0 7}$ & $\mathbf{0 . 0 0 8}$ & $\mathbf{0 . 0 0 8}$ & $\mathbf{0 . 0 1 8}$ & $\mathbf{0 . 0 4 1}$ & $\mathbf{0 . 0 3 6}$ & $\mathbf{0 . 0 1 0}$ \\
G3vsG2 & 0.469 & 0.460 & 0.467 & 0.419 & 0.519 & 0.686 & 0.838 & 0.990 & 0.056 & 0.168 \\
\hline
\end{tabular}

The bold numbers represent the statistically significant $p$-values $<0.05$

providing a perfusion insensitive ADC. Then the Image $\mathbf{J}$ software (http://rsb.info.nih.gov/ij/) was launched together with an in-house graphical user interface (GUI) that was developed in order to allow numerical assisted segmentation of the entire lesion volume and ADC histogram analysis. Since it is challenging to define the limits and boundaries of the tumors on ADC maps due to poor signal-to-noise ratio (SNR), the tumor volume was defined using the highest $b$ value, in our case $b=1000 \mathrm{~s} / \mathrm{mm}^{2} \mathrm{DW}$ images. The whole tumor was enclosed inside a user-defined bounding box in orthogonal views and automatically segmented by thresholding all the inner voxels with value over the mean $+0.5 \mathrm{SD}$. The resulting regions of interest (ROI) were then inspected and readjusted manually with an edit base validation protocol referring to $b=0 \mathrm{~s} / \mathrm{mm}^{2}$ diffusion-weighted images and TSE T2-weighted images, to encompass the totality of the PanNET lesion volume by avoiding adjacent pancreatic parenchyma within the ROIs and to exclude gross necrotic and cystic areas.

The ADC histogram was generated from the total tumor volume encompassed in all ROIs. The following quantitative parameters were derived: total volume, mean ADC (mADC), 5th percentile ADC (ADC5), 10th percentile ADC (ADC10), 25th percentile ADC (ADC25), 50th percentile ADC (ADC50), 75th percentile ADC (ADC75), 90th percentile ADC (ADC90) and 95th percentile ADC (ADC95), skewness and kurtosis.

\section{Statistical analysis}

Statistical analyses were performed with Stata software version 12.1 (Stata Corporation, College Station, TX).
Variables are reported as mean and standard deviation. $P$ values $<.05$ were considered to represent statistically significant differences. ANOVA with Tukey-Kramer post hoc comparisons for multiple pairwise comparisons [21,22] was performed to compare between histological tumor grades, age and gender, tumor volume, and histogram-derived ADC parameters.

Receiver operating characteristic (ROC) curves were obtained for each histogram-derived parameter. Optimal threshold for each histogram-derived parameter in differentiating histologic grades was considered to be the maximum value of Youden index [23].

\section{Results}

According to the ENETS grading system, 15/22 (68\%) tumors were classified as low grade (G1), 4/22 (18\%) as intermediate grade $(\mathrm{G} 2)$ and $3 / 22(14 \%)$ as high grade $(\mathrm{G} 3)$. Of the three patients that only had endoscopic biopsy, one had a G1 tumor and the other two had G3 tumors.

For histological grades G1, G2, and G3, the mean age was $54 \pm 14,55 \pm 17$, and $67 \pm 12$ years, respectively, and the gender was 9 males $/ 6$ females, 4 males $/ 0$ females, and 2 males/1 female, respectively. No statistically significant differences in age or gender were found between the three histological grades $(\mathrm{p}>.05)$.

Tumor volume ranged from 1.1 to $55 \mathrm{~mL}$. The mean volume for $\mathrm{G} 1$ was $2 \pm 3 \mathrm{~mL}$, for $\mathrm{G} 25 \pm 6 \mathrm{~mL}$ and $\mathrm{G} 3$ $55 \pm 15 \mathrm{~mL}$. The volume was statistically significantly higher in G3 compared to G1 and G2 $(p<0.0001)$.

Table 2 shows the quantitative histogram-derived parameters. Among these mADC, ADC75, ADC90, and ADC95 were statistically significantly different between 


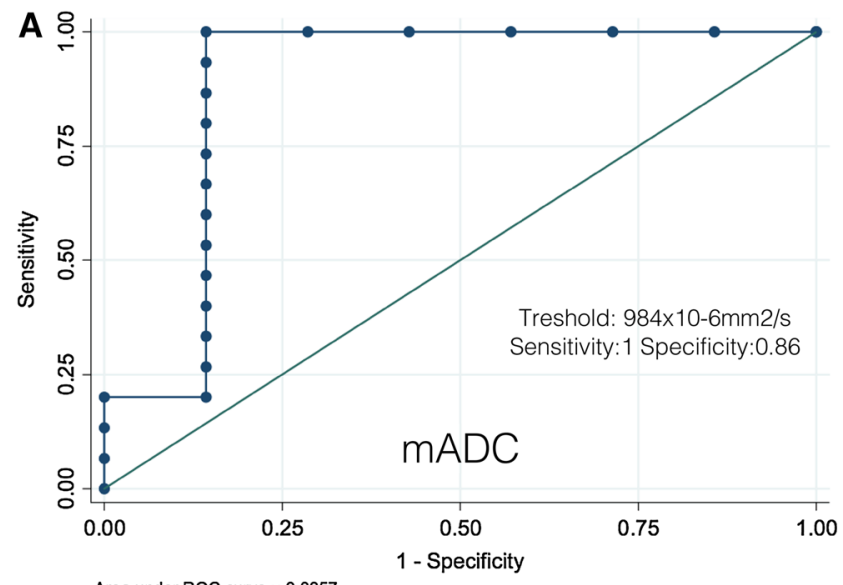

Area under ROC curve $=0.8857$

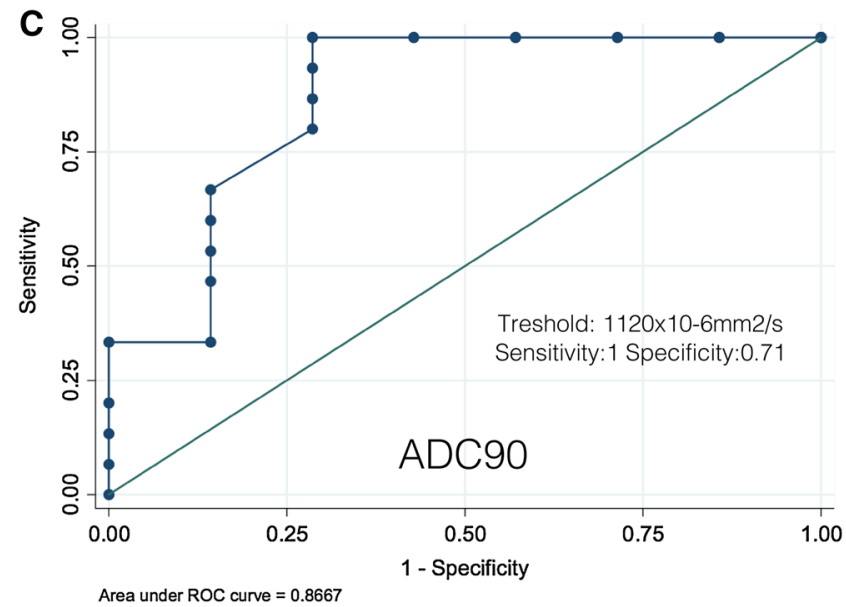

Fig. 1. ROC curve for A mADC, B ADC75, C ADC90, D ADC95 calculated for differentiating $G 1$ from G2/G3. The respective cut-off, sensitivity and specificity were $\mathbf{A}$

the three histological grades $(p=0.002 ; p=0.002$; $p=0.005 ; p=0.008)$. MeanADC, ADC75, ADC90, ADC95 were statistically significantly higher in G1 compared to G2 tumors. All histogram-derived parameters were statistically significantly higher in G1 than in G3 tumors. No statistically significant differences were found between $\mathrm{G} 2$ and $\mathrm{G} 3$ tumors. Table 3 gives the results of post hoc ANOVA pair analysis. Figure 1 represents ROC curves for the $\mathrm{mADC}$, ADC75, ADC90, and ADC95 calculated for differentiating G1 from G2/ G3. Skewness was positive in all tumor grades and statistically significantly higher in G3 $(1.01 \pm 1.140)$ compared to $\mathrm{G} 1 \quad(0.041 \pm 0.466 ; p<0.05)$ and to $\mathrm{G} 2$ $(0.065 \pm-0.387 ; p=0.056)$ tumors. Kurtosis increased with tumor grade and was significantly higher in G3 $(5.963 \pm 4.008)$ compared to G1 $(2.802 \pm 0.679)$ $(p=0.01)$. Representative images of G2 and G3 tumors are shown in Figs. 2 and 3, respectively.
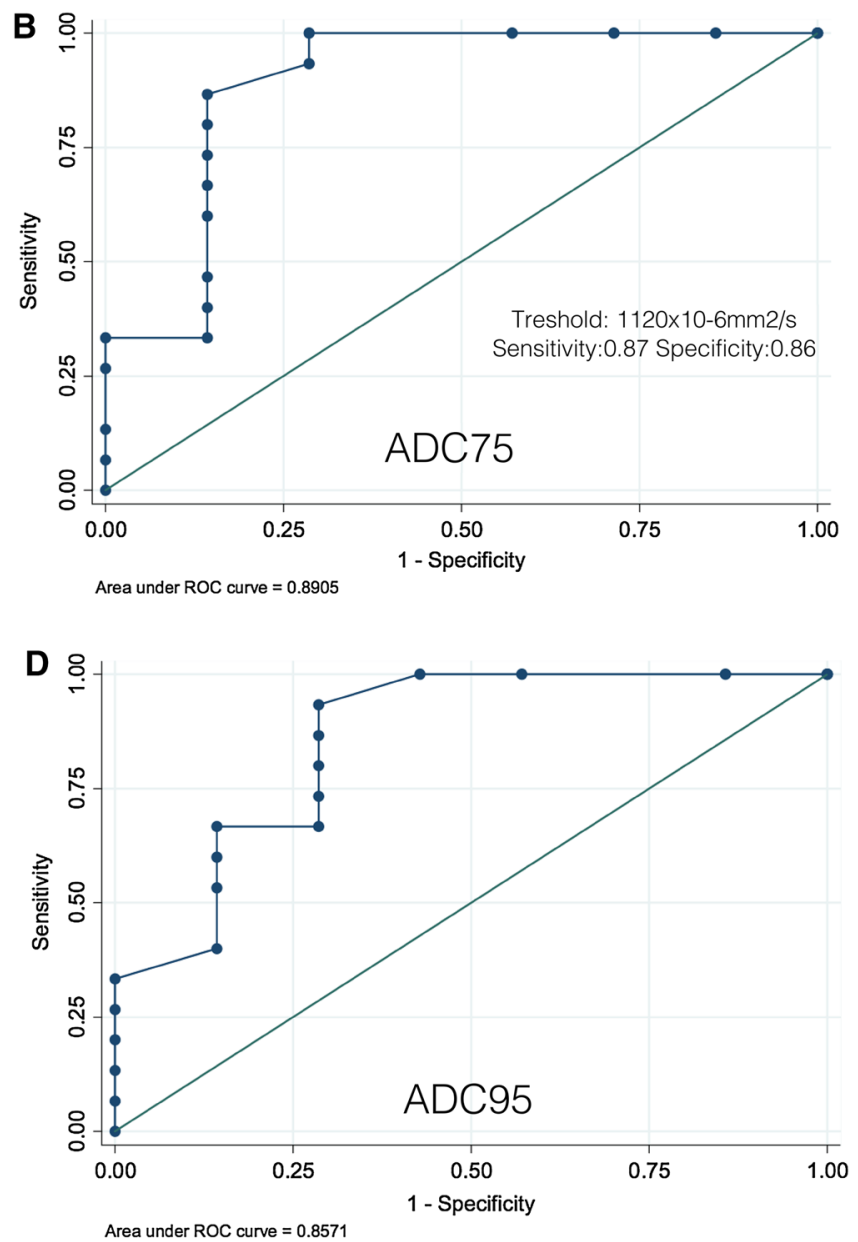

$984 \times 10^{-6} \mathrm{~mm}^{2} / \mathrm{s}, 100 \%, 86 \%$, B $1120 \times 10^{-6} \mathrm{~mm}^{2} / \mathrm{s}, 87 \%$, $86 \%$, C $1120 \times 10^{-6} \mathrm{~mm}^{2} / \mathrm{s}, 100 \%, 71 \%$, (D) $1184 \times 10^{-6}$ $\mathrm{mm}^{2} / \mathrm{s}, 93 \%, 71 \%$.

\section{Discussion}

The purpose of our study was to assess if ADC histogram-based analysis considering the entire tumor volume allows differentiation of the histological grades of PanNET. Previous studies have assessed the correlation between ADC and the histopathological characteristics of PanNETs, however, these were based on the evaluation of a single or few ROIs in the most representative sections of the tumor, giving limited information regarding the distribution of the ADCs. To our knowledge, there are no reports that have assessed PanNET histological grades with ADC histogram-based analysis considering the entire tumor volume. This method gives better information regarding the distribution of ADCs summarizing the diffusion pattern of water molecules within the whole tumor, also eliminating potential sampling bias. 

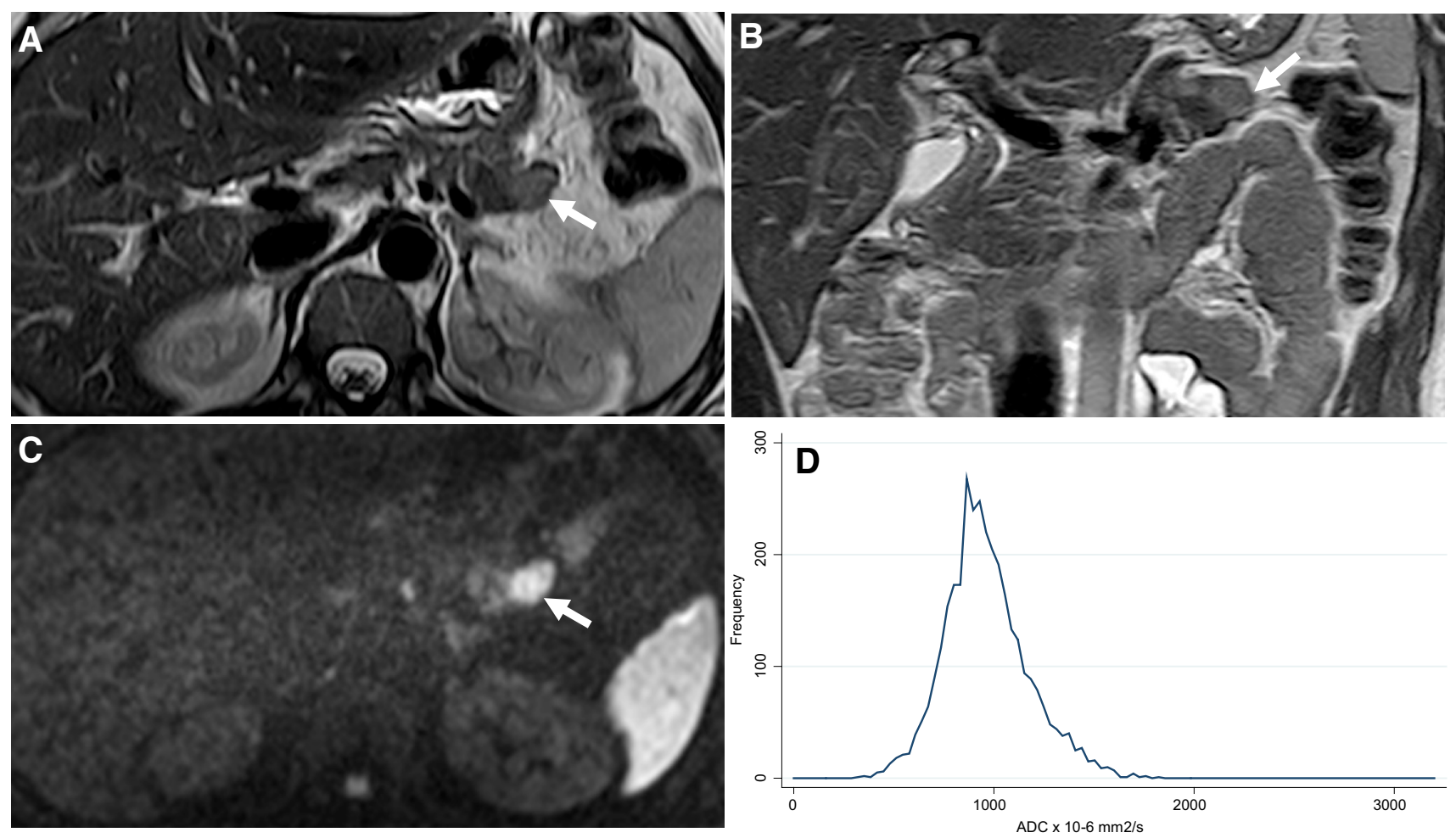

Fig. 2. Magnetic resonance images of a G2 PanNET from a 29-year-old female patient. Axial (A) and coronal (B) T2 weighted show a slight hyperintense exophytic lesion (arrow)

At histology, PanNETs are stroma-poor tumors with morphologic features that are dependent on their level of differentiation [5]. In WDNETs, which include G1 and G2 tumors, the cells are arranged as sheets of monotonous epithelial cells and have a fair amount of cytoplasm. The number of mitosis and the Ki-67 index allow the differentiation between G1 and G2. While G1 tumors show $<2$ mitosis per high power field (HPF), the G2 tumors may shown between 2 and 20 mitosis per HPF. The G3 tumors include the PDNEC, are divided into small and large-cell variants. While the large-cell variant is more common and characterized by large cells with prominent nucleoli and variable cytoplasmic volume, the small-cell variant shows high nucleus-to-cytoplasm ratio. Moreover, in G3 tumors the number of mitosis per HPF is $>20$ [5]. The increase of the mitotic index and the decrease of the cytoplasmic volume result in a decrease of the extracellular space with the consequent restriction of water molecules motion, resulting in a decrease in ADC as the tumor grade increases.

Similar to other previous studies, our results have shown that histogram-derived ADC parameters decrease as tumor grade increases. mADC, ADC75, ADC90, and ADC95 were statistically significantly higher in G1 compared to $\mathrm{G} 2(p<0.05)$ and that all histogram-derived parameters (mADC, ADC5, ADC10, ADC25, ADC50, ADC75, ADC90, ADC95) were statistically

in the pancreatic tail. Note tumor restriction on Axial DWI at $b=1000 \mathrm{~s} / \mathrm{mm}^{2}$ (C). D shows histogram distribution of ADC75 with a mean value of $975 \times 10^{-6} \mathrm{~mm}^{2} / \mathrm{s}$.

significantly higher in G1 tumors compared to G3 tumors $(p<0.05)$. Concerning G2 and G3 tumors, we found an overlap of ADC between these grades and we were not able to show a statistically significant difference. While this might be due to statistical chance, as our study could be underpowered by the small sample size (4 patients with G2 and 3 patients with G3 tumors), there may be an underlying histopathological reason. Despite the higher number of mitosis and nucleus-to-cytoplasma ratio, the G3 tumors may be characterized by intralesional necrosis [5], and this may impact the ADC values. Even if macroscopic necrotic and cystic areas were excluded from the ROIs, microscopic necrotic areas might have been included.

Despite the different classification, in the study of Wang et al., the mean ADC values of PanNET ranged from $770 \times 10^{-6} \mathrm{~mm}^{2} / \mathrm{s}$ (well differentiated endocrine carcinoma) to $2750 \times 10^{-6} \mathrm{~mm}^{2} / \mathrm{s}$ (well differentiated endocrine tumor with benign behaviour). Even though this analysis was based on a single ROI measurement, the results are similar to those of our study, as the mADC ranged from $474 \times 10^{-6} \mathrm{~mm}^{2} / \mathrm{s}$ (G3) to $1970 \times 10^{-6}$ $\mathrm{mm}^{2} / \mathrm{s}(\mathrm{G} 1)$. The lower values obtained in our study for mADC compared to the ones reported by Wang et al., can be explained by the fact that we included higher maximum $\mathrm{b}$-values and a higher minimum $b$ value $\left(b=150 \mathrm{~s} / \mathrm{mm}^{2}\right)$. 

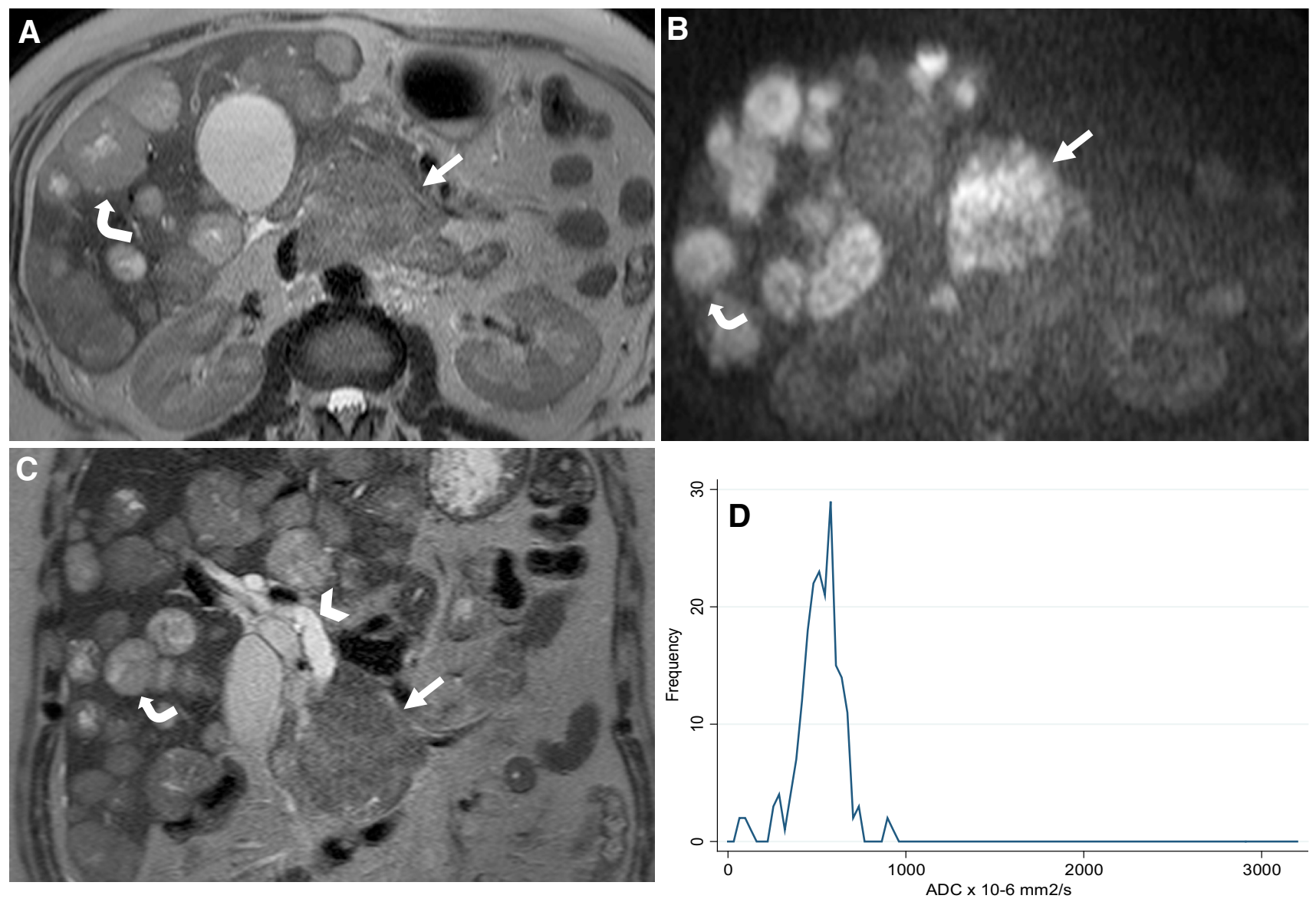

Fig. 3. Magnetic resonance images of a G3 PanNET from a 45-year-old male patient. Axial T2 weighted A shows a large heterogeneous hyperintense lesion (arrow) in the head, isthmus, and body of the pancreas. Also note nodular metastasis of the liver (curved arrow). Axial DWI at $b=1000 \mathrm{~s} / \mathrm{mm}^{2}$ B

The histogram-based analysis yields additional diffusion parameters regarding the distribution of ADCs, like skewness and kurtosis. Skewness is a measure of symmetry, or more precisely, the lack of symmetry. A distribution, or data set, is symmetric if it looks the same to the left and right of the center point. A positive skew indicates that the right tail of the distribution is fatter or longer than the left tail.

Kurtosis is a measure of whether the data are peaked or flat relative to a normal distribution. Data sets with high kurtosis tend to have a distinct peak near the mean, decline rather rapidly and have heavy tails [16].

In our results, we found that the skew was positive in all tumor grades but was statistically significantly higher in G3 $(1.01 \pm 1.140)$ compared to G1 $(0.041 \pm 0.466$; $p<0.05)$ and to $\mathrm{G} 2(0.065 \pm-0.387 ; p=0.056)$ tumors. This indicates that G3 tumors have a more asymmetric distribution when compared to G1 and G2 tumors. Moreover, we found that the kurtosis was statistically significantly higher in G3 (5.963 \pm 4.008$)$ when

shows marked restriction from the tumor (arrow) and from the hepatic metastases (curved arrow). Coronal T2-weighted image also shows dilatation of the extra-hepatic bile duct. D shows the histogram distribution of ADC75 with a mean value of $745 \times 10^{-6} \mathrm{~mm}^{2} / \mathrm{s}$.

compared to $\mathrm{G} 1 \quad(2.802 \pm 0.679 ; p<0.05)$ tumors. These results may reflect the marked histopathological heterogeneity of PDNEC included in the G3 group.

Among the limitations of this study, the sample size was small with only 4 patients in G2 and 3 patients in the G3 group. Indeed, we are dealing with relatively rare tumor. Further studies with larger sample size are required in order to increase the statistical power and build adequate predictive models. Furthermore, the reproducibility of the method was not assessed. Also 3 patients only had endoscopic biopsy specimens, one of which was classified as G1, therefore, we must acknowledge histopathology in this patient may be inaccurate due to sampling error from biopsy alone.

In conclusion, our results show that histogram-derived quantitative diffusion-weighted parameters may reflect the tumor structure and heterogeneity allowing the differentiation between $\mathrm{G} 1$ and $\mathrm{G} 2 / \mathrm{G} 3$ grade of PanNET. Further studies with larger sample size are required in order to build predictive models. 


\section{References}

1. Zhou C, Zhang J, Zheng Y, Zhu Z (2012) Pancreatic neuroendocrine tumors: a comprehensive review. Int J Cancer 131(5):10131022

2. Fraenkel M, Kim MK, Faggiano A, Valk GD (2012) Epidemiology of gastroenteropancreatic neuroendocrine tumours. Best Pract Res Clin Gastroenterol 26(6):691-703

3. Turaga KK, Kvols LK (2011) Recent progress in the understanding, diagnosis, and treatment of gastroenteropancreatic neuroendocrine tumors. CA Cancer J Clin 61(2):113-132

4. Piani C, Franchi GM, Cappelletti C, et al. (2008) Cytological Ki-67 in pancreatic endocrine tumours: an opportunity for pre-operative grading. Endocr Relat Cancer 15(1):175-181

5. Reid MD, Balci S, Saka B, Adsay NV (2014) Neuroendocrine tumors of the pancreas: current concepts and controversies. Endocr Pathol 25(1):65-79

6. Plockinger U, Rindi G, Arnold R, et al. (2004) Guidelines for the diagnosis and treatment of neuroendocrine gastrointestinal tumours. A consensus statement on behalf of the European Neuroendocrine Tumour Society (ENETS). Neuroendocrinology 80(6):394-424

7. Oberg K, Kvols L, Caplin M, et al. (2004) Consensus report on the use of somatostatin analogs for the management of neuroendocrine tumors of the gastroenteropancreatic system. Ann Oncol 15(6):966973

8. Pape UF, Jann H, Muller-Nordhorn J, et al. (2008) Prognostic relevance of a novel TNM classification system for upper gastroenteropancreatic neuroendocrine tumors. Cancer 113(2):256265

9. Yamaguchi T, Fujimori T, Tomita S, et al. (2013) Clinical validation of the gastrointestinal NET grading system: Ki67 index criteria of the WHO 2010 classification is appropriate to predict metastasis or recurrence. Diagn Pathol 8:65

10. Ramage JK, Ahmed A, Ardill J, et al. (2012) Guidelines for the management of gastroenteropancreatic neuroendocrine (including carcinoid) tumours (NETs). Gut 61(1):6-32

11. Schmid-Tannwald C, Oto A, Reiser MF, Zech CJ (2013) Diffusionweighted MRI of the abdomen: current value in clinical routine. J Magn Reson Imaging 37(1):35-47

12. Bakir B, Salmaslioglu A, Poyanli A, Rozanes I, Acunas B (2010) Diffusion weighted MR imaging of pancreatic islet cell tumors. Eur J Radiol 74(1):214-220
13. Wang Y, Chen ZE, Yaghmai V, et al. (2011) Diffusion-weighted MR imaging in pancreatic endocrine tumors correlated with histopathologic characteristics. J Magn Reson Imaging 33(5):10711079

14. Bull JG, Saunders DE, Clark CA (2012) Discrimination of paediatric brain tumours using apparent diffusion coefficient histograms. Eur Radiol 22(2):447-457

15. Kang Y, Choi SH, Kim YJ, et al. (2011) Gliomas: Histogram analysis of apparent diffusion coefficient maps with standard- or high-b-value diffusion-weighted MR imaging - correlation with tumor grade. Radiology 261(3):882-890

16. Ahn SJ, Choi SH, Kim YJ, et al. (2012) Histogram analysis of apparent diffusion coefficient map of standard and high B-value diffusion MR imaging in head and neck squamous cell carcinoma: a correlation study with histological grade. Acad Radiol 19(10): $1233-1240$

17. Hayashida Y, Hirai T, Morishita S, et al. (2006) Diffusion-weighted imaging of metastatic brain tumors: comparison with histologic type and tumor cellularity. AJNR Am J Neuroradiol 27(7):14191425

18. Heo SH, Shin SS, Kim JW, et al. (2013) Pre-treatment diffusionweighted MR imaging for predicting tumor recurrence in uterine cervical cancer treated with concurrent chemoradiation: value of histogram analysis of apparent diffusion coefficients. Korean $\mathbf{J}$ Radiol 14(4):616-625

19. Downey K, Riches SF, Morgan VA, et al. (2013) Relationship between imaging biomarkers of stage I cervical cancer and poorprognosis histologic features: quantitative histogram analysis of diffusion-weighted MR images. AJR Am J Roentgenol 200(2):314320

20. Brenner R, Metens T, Bali M, Demetter P, Matos C (2012) Pancreatic neuroendocrine tumor: added value of fusion of T2-weighted imaging and high $\mathrm{b}$-value diffusion-weighted imaging for tumor detection. Eur J Radiol 81(5):e746-e749

21. Dale BM, Braithwaite AC, Boll DT, Merkle EM (2010) Field strength and diffusion encoding technique affect the apparent diffusion coefficient measurements in diffusion-weighted imaging of the abdomen. Invest Radiol 45(2):104-108

22. Bender R, Lange S (2001) Adjusting for multiple testing - when and how? J Clin Epidemiol 54(4):343-349

23. Kumar R, Indrayan A (2011) Receiver operating characteristic (ROC) curve for medical researchers. Indian Pediatr 48(4):277-287 